\title{
Uso de máscaras de buceo modificadas para aplicación segura de ventilación no invasiva. Estudio piloto
}

\author{
Modified snorkeling facemasks for safe application of non-invasive ventilation. A pilot study \\ Uso de máscaras de mergulho modificadas para aplicação segura de ventilação não \\ invasiva. Estudo piloto
}

\author{
Rodrigo Beltramelli, Pedro Alzugaray², Nicolás Rucks³, Cristina Santos ${ }^{4}$, \\ Carolina Poradosu ${ }^{5}$, Gimena González ${ }^{6}$, Ana Musetti ${ }^{7}$, Martín Angulo8
}

\section{Resumen}

Introducción: en el contexto de la pandemia de SARS-CoV-2, diferentes autores propusieron adaptaciones a máscaras de buceo comerciales (máscaras modificadas, MM) para proporcionar oxigenoterapia a pacientes con o sin presión positiva. Hasta la fecha, ninguno ha evaluado su desempeño como interfaz para la ventilación no invasiva (VNI) en el modo de soporte de presión inspiratoria (PSI).

Objetivos: desarrollar una interfaz de VNI utilizando MM y evaluar su rendimiento en comparación con una máscara oronasal (MC) convencional.

Métodos: las MM se adaptaron como interfaces VNI utilizando dos piezas creadas mediante impresión 3D. Se comparó su desempeño contra una MC en 10 voluntarios sanos en modo PSI $\left(3 \mathrm{cmH}_{2} \mathrm{O}\right)$ con dos niveles de presión positiva al final de la espiración (PEEP 4 y $8 \mathrm{cmH}_{2} \mathrm{O}$ ). Se compararon: fugas del sistema, frecuencia respiratoria, volumen corriente, oximetría de pulso, $\mathrm{CO}_{2}$ transcutáneo y comodidad.

Resultados: con $4 \mathrm{cmH}_{2} \mathrm{O}$ de PEEP no hubo diferencias significativas entre las máscaras en ninguna de las variables estudiadas. Con $8 \mathrm{cmH}_{2} \mathrm{O}$ de PEEP, el uso de MM se asoció con un menor nivel de $\mathrm{CO}_{2}$ tc $(41,0 \pm 5,7$ vs 43,5 $\pm 8,1 \mathrm{mmHg} ; p=0,03)$ y un mayor confort $(8,3 \pm 1,1$ vs $6,5 \pm 1,4 ; p<0,01)$ que las $M C$.

Conclusiones: el uso de MM para realizar VNI presenta, en voluntarios sanos, un rendimiento similar a las mascarillas oronasales estándar, con la ventaja de mayor comodidad y menor costo. Si bien restan estudios para avalar su utilización, en la emergencia sanitaria provocada por la pandemia SARS-CoV-2, la adaptación de máscaras de buceo podría representar una opción válida frente a la escasez de interfaces comerciales.

\footnotetext{
1. Asistente Laboratorio Exploración Funcional Respiratorio, Centro de Tratamiento Intensivo, Hospital de Clínicas, Montevideo, Uruguay. 2. Profesor Agregado Laboratorio Exploración Funcional Respiratorio, Centro de Tratamiento Intensivo, Hospital de Clínicas, Montevideo, Uruguay. 3. Asistente Área Proyectual, Perfil Producto de la Escuela Universitaria Centro de Diseño, Subárea Representación - Modelado 3D Para Prototipado. Ayudante Área Tecnológica, Perfil Producto de la Escuela Universitaria Centro de Diseño, Subárea Tecnología. Universidad de la República, Montevideo, Uruguay.

4. Ex-Profesora Agregada Laboratorio Exploración Funcional Respiratorio, Centro de Tratamiento Intensivo, Hospital de Clínicas, Montevideo, Uruguay. 5. Directora de la Escuela Universitaria Centro de Diseño, Profesora Adjunta Área Proyectual de la Escuela Universitaria Centro de Diseño/FADU- Universidad de la República, Montevideo, Uruguay.

6. Licenciada Neumocardióloga, Laboratorio Exploración Funcional Respiratorio. Hospital de Clínicas, Montevideo, Uruguay

7. Profesora Agregada Laboratorio Exploración Funcional Respiratorio. Cátedra de Neumología, Universidad de la República, Montevideo, Uruguay.

8. Profesor Agregado Departamento de Fisiopatología, Facultad de Medicina, Universidad de la República. Profesor Adjunto Laboratorio Exploración Funcional Respiratorio, Centro de Tratamiento Intensivo, Hospital de Clínicas, Montevideo, Uruguay

Laboratorio Exploración Funcional Respiratorio, Cátedra de Medicina Intensiva, Universidad de la República, Montevideo, Uruguay.

Correspondencia: Dr. Rodrigo Beltramelli. Correo electrónico: rodrigobeltramelli@gmail.com

Financiamiento: ninguno

El protocolo de investigación fue aprobado por el Comité de Ética del Hospital de Clínicas.

Recibido: $2 / 3 / 2021$

Aprobado: 9/8/2021

Attribution-NonCommercial 4.0 International (CC BY-NC 4.0)
} 
Palabras clave: Ventilación no invasiva

Máscaras de buceo

COVID-19

Key words: $\quad$ Noninvasive ventilation

Diving masks

COVID-19

\section{Introducción}

La ventilación no invasiva (VNI) consiste en la administración de ventilación mecánica sin necesidad de una vía aérea artificial. Es ampliamente utilizada para el tratamiento de pacientes con insuficiencia respiratoria de diferentes causas ${ }^{(1)}$. Las claves para el éxito de la VNI son la selección cuidadosa de pacientes y la elección del soporte ventilatorio adecuado para cada uno. En el mismo sentido, es fundamental la elección de una interfaz óptima y cómoda, que asegure un correcto sellado para minimizar las fugas sin provocar lesiones en el rostro del paciente. Existen varios tipos de interfases para realizar VNI, las más utilizadas en la práctica clínica en las Unidades de Cuidados Intensivos (UCI) son las máscaras faciales (u oronasales), las cuales presentan una incidencia de lesiones de apoyo que puede alcanzar hasta el $20 \%$, principalmente a nivel de tabique nasal ${ }^{(2)}$. La máscara facial total es otro tipo de interfaz con resultados similares a la máscara oronasal sin tanta incidencia de lesiones de apoyo, pero con la desventaja de presentar mayor fuga aérea y mayor espacio muerto ${ }^{(3)}$.

Con la emergencia de la pandemia de SARS-CoV-2, la escasez de insumos y equipamiento médico y el costo de los mismos desencadenó la búsqueda de alternativas innovadoras que permitieran brindar soporte respiratorio a todos los pacientes ${ }^{(4)}$. En este sentido, distintos autores han propuesto adaptaciones a máscaras de buceo comerciales que permitirían brindar oxigenoterapia con o sin presión positiva a los pacientes, o incluso emplearse como equipo de protección para el personal sanitario $^{(5-11)}$.

Con determinadas modificaciones, las máscaras de buceo podrían constituir una interfaz adecuada para realizar VNI en las UCI. Si bien se ha descrito su utilización con válvulas descartables de presión positiva al final de la espiración (PEEP) o equipos portátiles de presión positiva continua en vía aérea $(\mathrm{CPAP})^{(12)}$, no se ha evaluado su desempeño como interfaz para VNI empleando presión de soporte inspiratoria (PS) y PEEP mediante un equipo de ventilación mecánica de alta gama como los que se utilizan en las UCI.

El objetivo del presente trabajo fue desarrollar una interfaz para VNI a partir de máscaras de buceo comer- ciales y evaluar su desempeño en comparación con una máscara oronasal estándar.

\section{Metodología}

En conjunto entre el Laboratorio de Exploración Funcional Respiratorio del Hospital de Clínicas y la Escuela Universitaria Centro de Diseño (UDELAR) se trabajó en las modificaciones para transformar una máscara de buceo en una interfaz adecuada y segura para ventilación no invasiva. Utilizando máscaras de buceo comerciales (Free Breath M2068G®) y mediante impresión $3 \mathrm{D}$, se confeccionaron dos piezas, una para el sellado de la abertura superior de la máscara (pieza \#1) y otra ubicada en la abertura frontal de la máscara para la conexión de la pieza en Y del ventilador (pieza \#2), con o sin interposición de un filtro HEPA (figura 1). Para comparar el área de intercambio de gases se realizó la medición del volumen de cada máscara mediante técnica de desplazamiento de líquidos, arrojando que ambas interfaces, en su área de intercambio de gases tienen volúmenes similares (aproximadamente $200 \mathrm{ml}$ ). En esta etapa se buscó priorizar los aspectos de comodidad para su uso, hermeticidad de los acoples y facilidad de armado. Los materiales utilizados permiten la desinfección de las máscaras mediante el mismo protocolo empleado en las máscaras convencionales de VNI.

La posición del acople en la parte frontal de la máscara frente a la alternativa de mantener el flujo de gases en la sección superior aumenta el confort debido a la disposición de la conexión hacia el ventilador, disminuyendo además la deposición de humedad en la sección del visor de la máscara producto del flujo exhalado.

Una vez lograda la modificación de las máscaras, se procedió a su validación mediante la comparación de su desempeño contra una máscara oronasal convencional (FreeMotion ${ }^{\circledR}$ RT041, Fisher \& Paykel Healthcare, Nueva Zelanda) en 10 voluntarios sanos en modalidad PSI con dos niveles de PEEP.

Todo el proceso de comparación se realizó utilizando un ventilador Servo-i ${ }^{\circledR}$ (Maquet Critical Care, Solna, Suecia), con una fracción inspirada de oxígeno programada de 0,21 y una presión de soporte constante de 3 $\mathrm{cmH}_{2} \mathrm{O}$. En todos los casos se fijó el trigger inspiratorio en $-2 \mathrm{cmH}_{2} \mathrm{O}$, y el ciclado de inspiración a espiración en $25 \%$. Se compararon las máscaras convencionales (MC) de VNI con las máscaras de buceo modificadas (MM) en 2 niveles de PEEP $\left(4\right.$ y $\left.8 \mathrm{cmH}_{2} \mathrm{O}\right)$ durante 5 minutos en cada condición. Durante todo el procedimiento se registraron en cada minuto: nivel de fuga, frecuencia respiratoria y volumen corriente medido por el equipo de ventilación mecánica, oximetría de pulso $\left(\mathrm{SpO}_{2}\right)$ y medición continua de $\mathrm{CO}_{2}$ transcutáneo $\left(\mathrm{CO}_{2} \mathrm{tc}\right)$ a través de un monitor transcutáneo de gases (SenTec Digital Monito- 

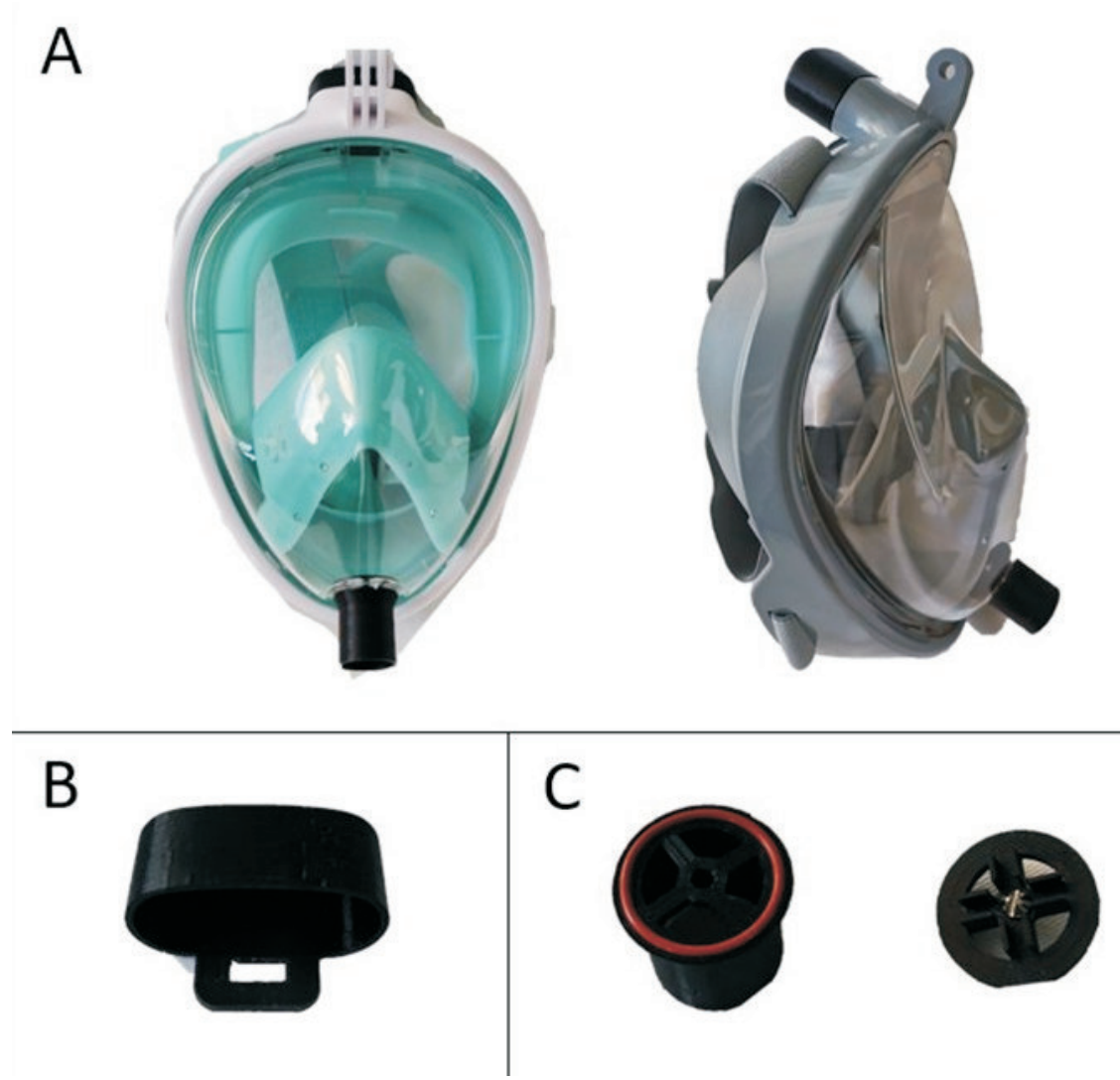

Figura 1. Máscaras de buceo modificadas. A. Máscaras de buceo modificadas para utilización en VNI. B: Pieza \#1 para ocluir la entrada superior de la máscara. C: Pieza \#2 para conexión a la pieza en Y del circuito de ventilador.

ring System $\left.{ }^{\circledR}\right)$ y para valorar el confort se utilizó una escala de 1-10, donde 1 fue menos confortable y 10 más confortable.

El protocolo de investigación fue aprobado por el Comité de Ética del Hospital de Clínicas.

\section{Análisis estadístico}

Los resultados se expresan como media \pm desvío estándar. La comparación se realizó con t-student o test de Wilcoxon según correspondiera. Se consideró estadísticamente significativa una $p<0,05$. Los datos se analizaron con el software GraphPad Prism versión 8.4.3.

\section{Resultados}

Con niveles de PEEP de $4 \mathrm{cmH}_{2} \mathrm{O}$, las $\mathrm{MC}$ presentaron un nivel de fuga de $23,1 \pm 16,1 \%$ vs $35,9 \pm 17,8 \%$ (p $=$ $0,06)$ de las MM. La frecuencia respiratoria de los participantes fue de 14,8 $\pm 3,0 \mathrm{rpm}$ con las $\mathrm{MC}$ vs 13,8 $\pm 2,5$ rpm con las MM (p=0,08); el volumen corriente administrado con las MC fue de $830 \pm 248 \mathrm{ml}$ vs $681 \pm 267$ $\mathrm{ml}(\mathrm{p}=0,20)$ con las MM. No hubo diferencias en la $\mathrm{SpO}_{2}$ de los voluntarios durante la ventilación con máscaras convencionales o modificadas $(98 \pm 1 \%$ vs $98 \pm$
$1 \%$, respectivamente, $\mathrm{p}=0,40$ ); así como tampoco en la $\mathrm{CO}_{2}$ tc $(43,9 \pm 7,8 \mathrm{mmHg}$ vs $41,7 \pm 6,6 \mathrm{mmHg}$, respectivamente, $p=0,14)$. Los niveles de confort reportados por los participantes durante el uso de las $\mathrm{MC}$ con 4 $\mathrm{cmH}_{2} \mathrm{O}$ de PEEP fue de $8,0 \pm 1,2$, mientras que con las MM fue de 8,7 $\pm 0,9(p=0,06)$ (figura 2).

Cuando el nivel de PEEP se fijó en $8 \mathrm{cmH}_{2} \mathrm{O}$, la fuga aérea fue de $32,0 \pm 19,8 \%$ con las MC vs $47,6 \pm 21,7 \%$ con las MM $(p=0,15)$. No hubo diferencias estadísticamente significativas en la frecuencia respiratoria $(14,2 \pm$ $3,4 \mathrm{rpm}$ vs $13,9 \pm 3,0 \mathrm{rpm}, \mathrm{p}=0,70$ ), volumen corriente administrado ( $804 \pm 225 \mathrm{ml}$ vs $772 \pm 229 \mathrm{ml}, \mathrm{p}=0,50)$ y $\mathrm{SpO}_{2}(98 \pm 1 \%$ vs $98 \pm 2 \%, \mathrm{p}=0,18)$ observadas con máscaras convencionales o modificadas, respectivamente. El $\mathrm{CO}_{2}$ tc con las $\mathrm{MC}$ fue de 43,5 $\pm 8,1 \mathrm{mmHg}$ vs $41,0 \pm 5,7 \mathrm{mmHg}$ con las MM ( $\mathrm{p}=0,03)$. Finalmente, los participantes reportaron un nivel de confort significativamente mayor con las máscaras modificadas que con las convencionales $(8,3 \pm 1,1$ vs $6,5 \pm 1,4, p<0,01)$ (figura 3).

El nivel de presurización con todas las interfases y condiciones estudiadas fue el adecuado, sin variar sustancialmente con el seteado por los investigadores. 


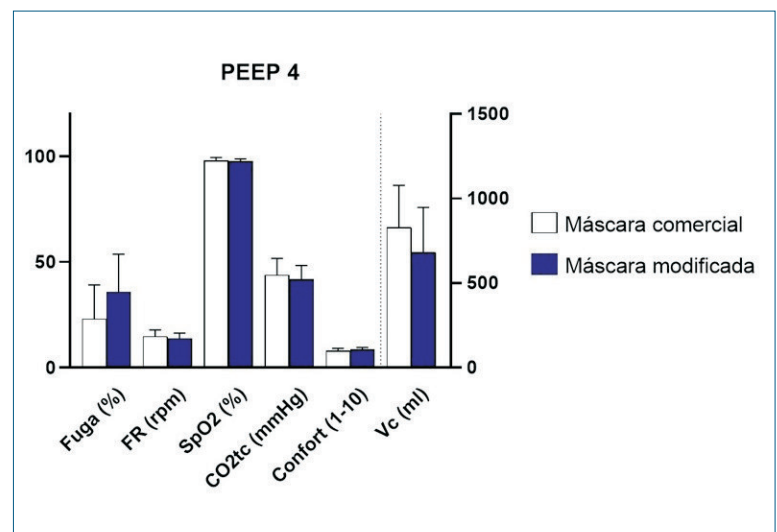

Figura 2. Resultados comparativos entre las máscaras comerciales (barras blancas) y las máscaras modificadas (barras azules) con $4 \mathrm{cmH}_{2} \mathrm{O}$ de PEEP No se encontraron diferencias estadísticamente significativas en ninguna de las variables estudiadas.

\section{Discusión}

En términos generales en voluntarios sanos, la VNI con máscaras de buceo modificadas fue posible, bien tolerada y con un nivel de desempeño similar a las máscaras convencionales. Con niveles de PEEP de 4 $\mathrm{cmH}_{2} \mathrm{O}$ no se encontraron diferencias significativas en ninguna de las variables analizadas, mientras que con niveles de PEEP de $8 \mathrm{cmH}_{2} \mathrm{O}$ se observaron diferencias significativas en los niveles de $\mathrm{CO}_{2}$ tc y en confort a favor de las MM. Se destaca, con este nivel de PEEP, la presencia de mayor porcentaje de fugas con las MM comparado con las $\mathrm{MC}$, aunque esto no fue estadísticamente significativo.

En el análisis individual de las variables, a diferencia de lo esperado, hubo una tendencia a mayor fuga con las MM. Esto puede deberse a que las máscaras de buceo fueron creadas para soportar presión desde fuera de la máscara hacia el rostro del paciente, empujando la misma y asegurando un correcto sellado; en el caso de la VNI, la presión administrada al interior de la máscara aleja la misma del rostro del paciente. Esta diferencia podría explicar la presencia de fugas mayores en las MM comparado con las máscaras comerciales. En cuanto al funcionamiento del sistema ventilo-respiratorio, no encontramos interferencia con su normal funcionamiento en ambas interfases. La frecuencia respiratoria y los volúmenes corrientes no variaron significativamente entre las MC y las MM, independientemente del nivel de PEEP utilizado. La capacidad de mantener el nivel de oxigenación fue similar para ambas máscaras.

El menor nivel de $\mathrm{CO}_{2}$ tc con niveles de PEEP de 8 $\mathrm{cmH}_{2} \mathrm{O}$ de las MM pensamos que probablemente sea se-

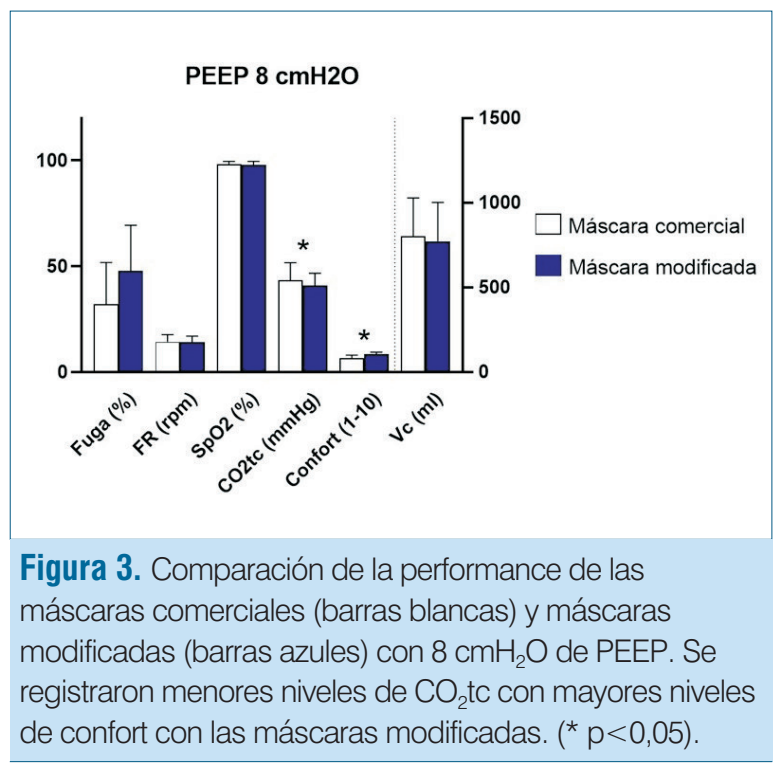

cundario a la compensación ventilatoria que presentan los voluntarios sanos frente a la pérdida de presurización debido al mayor porcentaje de fugas con estas interfaces, aunque el mismo no haya sido estadísticamente significativo.

Los niveles de confort con niveles bajos de PEEP (4 $\mathrm{cmH}_{2} \mathrm{O}$ ), fueron elevados y similares en ambas interfaces, seguramente debido a los bajos niveles de presurización utilizados. En cambio, cuando aumentamos la PEEP a $8 \mathrm{cmH}_{2} \mathrm{O}$, se registraron fugas aéreas hacia los ojos de los participantes, lo que obligó a revisar los ajustes de los arneses, y debido a que el ajuste de los arneses de las MM es más confortable, y el aro de silicona que contacta con el rostro del paciente es más amplio y permite distribuir la presión ejercida en más puntos de apoyo, hacen que se pueda ejercer una presión mayor con los arneses sin aumentar el disconfort. En cambio, con las MC, para evitar fugas con niveles superiores de presurización, se hace necesario ajustar más los arneses de sujeción, aumentando la presión de la máscara contra en tabique nasal para evitar fugas hacia los ojos, lo cual hace que el confort disminuya.

\section{Conclusiones}

La utilización de MM para realización de VNI presenta, en voluntarios sanos, un desempeño similar a las MC disponibles en el mercado, con la ventaja de presentar mayor confort y menor costo. Si bien aún faltan estudios para avalar su utilización en pacientes con patología respiratoria asociada, en el marco de la emergencia sanitaria por la pandemia causada por SARS-Cov-2, la adaptación de máscaras de buceo podría representar una opción válida frente a la escasez de interfaces comerciales. 


\section{Abstract}

Introduction: within the context of the SARS-CoV-2 pandemic, different authors proposed adaptations to snorkeling masks available in the market (modified masks: MM) to provide oxygen therapy with positive pressure ventilation or not. Until today, none of them has assessed its performance as an interface for non-invasive ventilation (NIV) in the inspiratory pressure support mode.

Objective: to develop an interface of NIV using $\mathrm{MM}$ and assess its performance with a conventional full mask.

Method: the MM were adapted as NIV interfaces using two pieces created by $3 \mathrm{D}$ printing. Their performance was compared to a that of a conventional mask in 10 healthy volunteers in inspiratory pressure support (3 $\mathrm{cmH}_{2} \mathrm{O}$ ) mode with two levels of positive pressure towards the end of expiration (PEEP 4 and $8 \mathrm{cmH}_{2} \mathrm{O}$ ). The following were compared: system leaks, respiratory rate, normal volume, pulse oximetry, transcutaneous carbon dioxide and comfort.

Results: with $4 \mathrm{cmH}_{2} \mathrm{O}$ of PEEP, no significant differences were found between masks in none of the variables studied. With $8 \mathrm{cmH}_{2} \mathrm{O}$ de PEEP, the use of MM were associated to a lower level of $\mathrm{CO}_{2}$ tc $(41.0 \pm 5.7 \mathrm{vs}$ $43.5 \pm 8.1 \mathrm{mmHg} ; \mathrm{p}=0.03)$ and greater comfort $(8.3 \pm 1.1$ vs $6.5 \pm 1.4 ; \mathrm{p}<0.01)$ than conventional masks $(\mathrm{CM})$.

Conclusions: in healthy volunteers, MM for NIV evidence a similar performance than that of standard oronasal masks, and have a further advantage, as they are more comfortable and cheaper. Even if further studies are needed to support its use, modified snorkeling masks could represent a valid option during the health emergency caused by the SARS-CoV-2 pandemic in consideration of the scarce interfaces available in the market.

\section{Resumo}

Introdução: no contexto da pandemia de SARS-CoV2, diferentes autores propuseram adaptações às máscaras de mergulho comerciais (máscaras modificadas, MM) para fornecer oxigenoterapia a pacientes com ou sem pressão positiva. Até o momento, nenhum avaliou seu desempenho como interface para ventilação não invasiva (VNI) no modo de suporte de pressão inspiratória (PSI).

Objetivos: desenvolver uma interface de VNI usando MM e avaliar seu desempenho em comparação com uma máscara oronasal convencional (MC).

Métodos: as MMs foram adaptadas como interfaces VNI usando duas peças criadas por impressão 3D. Seu desempenho contra uma MC foi comparado em 10 vo- luntários saudáveis no modo PSI $\left(3 \mathrm{cmH}_{2} \mathrm{O}\right)$ com dois níveis de pressão positiva no final da expiração (PEEP 4 e $8 \mathrm{cmH}_{2} \mathrm{O}$ ). Vazamentos do sistema, frequência respiratória, volume corrente, oximetria de pulso, $\mathrm{CO}_{2}$ transcutâneo e conforto foram comparados.

Resultados: com $4 \mathrm{cmH}_{2} \mathrm{O}$ de PEEP, não se observaram diferenças significativas entre as máscaras em nenhuma das variáveis estudadas. Com PEEP de 8 $\mathrm{cmH}_{2} \mathrm{O}$, o uso de $\mathrm{MM}$ foi associado a um menor nível de $\mathrm{CO}_{2} \mathrm{tc}(41,0 \pm 5,7$ vs $43,5 \pm 8,1 \mathrm{mmHg} ; \mathrm{p}=0,03)$ e maior conforto $(8,3 \pm 1,1$ vs $6,5 \pm 1,4 ; \mathrm{p}<0,01)$ do que a MC.

Conclusões: o uso do MM para a realização da VNI apresenta, em voluntários saudáveis, desempenho semelhante às máscaras oronasais padrão, com a vantagem de maior conforto e menor custo. Embora ainda sejam necessários mais estudos que embasem seu uso, na emergência sanitária ocasionada pela pandemia do SARS-CoV-2, a adaptação de máscaras de mergulho pode representar uma opção válida diante da escassez de interfaces comerciais.

\section{Bibliografía}

1. Hill N, Brennan J, Garpestad E, Nava S. Noninvasive ventilation in acute respiratory failure. Crit Care Med 2007; 35(10):2402-7.

2. Gregoretti $\mathbf{C}$, Confalonieri M, Navalesi P, Squadrone $\mathbf{V}$, Frigerio P, Beltrame F, et al. Evaluation of patient skin breakdown and comfort with a new face mask for non-invasive ventilation: a multi-center study. Intensive Care Med 2002; 28(3):278-84.

3. Belchior I, Gonçalves M, Winck J. Continuous noninvasive ventilation delivered by a novel total face mask: a case series report. Respir Care 2012; 57(3):449-53.

4. Pérez-Mañanes R, García de San José S, Desco-Menéndez M, Sánchez-Arcilla I, González-Fernández E, Vaquero-Martín J, et al. Point-of-care 3D printing during the COVID-19 pandemic. What role does the manufacturing university hospital play? Res Sq 2020; 1:1-17. doi: 10.21203/rs.3.rs-34063/v1.

5. Germonpre P, Van Rompaey D, Balestra C. Evaluation of Protection level, respiratory safety, and practical aspects of commercially available snorkel masks as personal protection devices against aerosolized contaminants and SARS-CoV2. Int J Environ Res Public Health 2020; 17(12):4347. doi: 10.3390/ijerph17124347.

6. Greig P, Carvalho C, El-Boghdadly K, Ramessur S. Safety testing improvised COVID-19 personal protective equipment based on a modified full-face snorkel mask. Anaesthesia 2020; 75(7):970-1.

7. Guan L, Zhou L, Zhang J, Peng W, Chen R. More awareness is needed for severe acute respiratory syndrome coronavirus 2019 transmission through exhaled air during non-inva- 
sive respiratory support: experience from China. Eur Respir J 2020; 55(3):2000352. doi: 10.1183/13993003.00352-2020.

8. Hui D, Chow B, Lo T, Tsang O, Ko F, Ng S, et al. Exhaled air dispersion during high-flow nasal cannula therapy versus CPAP via different masks. Eur Respir J 2019; 53(4):1802339. doi: 10.1183/13993003.02339-2018.

9. Kechli M, Lerman J, Ross M. Modifying a full-face snorkel mask to meet $\mathrm{n} 95$ respirator standards for use with coronavirus disease 2019 patients. A A Pract 2020; 14(7):e01237. doi: 10.1213/XAA.0000000000001237.

10. Vicini C, Cammaroto G, Meccariello G, Iannella G, Fragale $\mathbf{M}$, Cacco $\mathbf{T}$, et al. Overview of different modified full-face snorkelling masks for intraoperative protection. Acta Otorhinolaryngol Ital 2020; 40(5):317-24. doi: 10.14639/0392-100X-N0841.

11. Thierry B, Célérier C, Simon F, Lacroix C, Khonsari R. How and why use the EasyBreath ${ }^{\circledR}$ surface snorkeling mask as a personal protective equipment during the COVID-19 pandemic? Eur Ann Otorhinolaryngol Head Neck Dis 2020; 137(4):329-31. doi: 10.1016/j.anorl.2020.05.006.

12. Landry S, Mann D, Djumas L, Messineo L, Terrill P, Thomson L, et al. Laboratory performance of oronasal CPAP and adapted snorkel masks to entrain oxygen and CPAP. Respirology 2020; 25(12):1309-12.

\section{Contribución de autores}

Rodrigo Beltramelli: ORCID 0000-0001-8707-1570. Diseño, ejecución, análisis, interpretación de resultados, redacción.

Pedro Alzugaray: ORCID 0000-0002-3165-1493. Ejecución, análisis, interpretación de resultados, redacción, revisión crítica.

Nicolás Rucks: ORCID 0000-0003-0375-9357. Concepción, diseño, ejecución, redacción.

Cristina Santos: ORCID 0000-0003-0084-6412. Análisis, interpretación de resultados, revisión crítica.

Carolina Poradosu: ORCID 0000-0002-7810-1800. Ejecución, redacción, revisión crítica.

Gimena González: ORCID 0000-0001-8016-1290. Ejecución, análisis, interpretación de resultados.

Ana Musetti: ORCID 0000-0003-3014-7591. Ejecución, análisis, revisión crítica.

Martín Angulo: ORCID 0000-0002-3326-5764. Concepción, diseño, ejecución, análisis, interpretación de resultados, redacción, revisión crítica. 